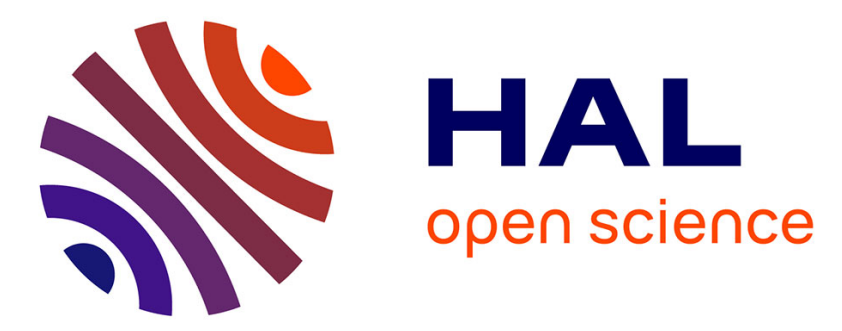

\title{
Effect of local stress heterogeneities on dislocation fields: Examples from transient creep in polycrystalline ice
}

\author{
S. Piazolo, M. Montagnat, F. Grennerat, H. Moulinec, J. Wheeler
}

\section{To cite this version:}

S. Piazolo, M. Montagnat, F. Grennerat, H. Moulinec, J. Wheeler. Effect of local stress heterogeneities on dislocation fields: Examples from transient creep in polycrystalline ice. Acta Materialia, 2015, 90, pp.303-309. 10.1016/j.actamat.2015.02.046 . hal-01746106

\section{HAL Id: hal-01746106 \\ https://hal.science/hal-01746106}

Submitted on 29 Mar 2018

HAL is a multi-disciplinary open access archive for the deposit and dissemination of scientific research documents, whether they are published or not. The documents may come from teaching and research institutions in France or abroad, or from public or private research centers.
L'archive ouverte pluridisciplinaire HAL, est destinée au dépôt et à la diffusion de documents scientifiques de niveau recherche, publiés ou non, émanant des établissements d'enseignement et de recherche français ou étrangers, des laboratoires publics ou privés. 


\title{
Effect of local stress heterogeneities on dislocation fields: Examples from transient creep in polycrystalline ice.
}

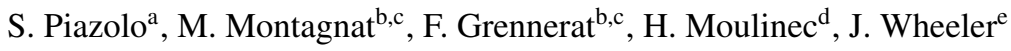 \\ ${ }^{a}$ ARC Center of Excellence for Core to Crust Fluid Systems (CCFS) and GEMOC, Department of Earth and Planetary Science, Macquarie \\ University, NSW 2109, Australia \\ ${ }^{b}$ CNRS, LGGE, UMR5183, 54 rue Molière, 38041 Grenoble, France \\ ${ }^{c}$ Univ. Grenoble Alpes, LGGE, 38041 Grenoble, France \\ ${ }^{d}$ Laboratoire de Mécanique et dAcoustique, CNRS, UPR7051, Aix Marseille University, Centrale Marseille, 31 Chemin Joseph Aiguier, 13402 \\ Marseille Cedex 20, France \\ ${ }^{e}$ Department of Earth and Ocean Sciences, School of Environmental Science, Liverpool University, Liverpool L69 3GP, UK
}

\begin{abstract}
This work presents a coupled experimental and modeling approach to better understand the role of stress field heterogeneities on deformation behavior in material with a high viscoplastic anisotropy e.g. polycrystalline ice. Full-field elasto-viscoplastic modeling is used to predict the local stress and strain field during transient creep in a polycrystalline ice sample. Modeling input includes the experimental starting microstructure and a validated slip system dependent flow law. EBSD measurements on selected areas are used to estimate the local dislocation field utilizing the Weighted Burgers Vector (WBV) analysis. Areas of local stress concentration correlate with triple junctions and grain boundaries, originating from strain incompatibilities between differently oriented grains. In these areas of highly heterogeneous stress patterns, (a) kink bands are formed and (b) WBV analysis shows a non-negligible c-axis component of the WBV. The correlation between this defect structure and presence of kink bands suggest that kink band formation is an efficient accommodation deformation mode.
\end{abstract}

This is the author version of the paper, find the editor one at http://dx.doi.org/10.1016/j.actamat.2015.02.046

Keywords:

stress heterogeneities, dislocation field, electron backscatter diffraction, full-field modeling, kink bands, viscoplastic anisotropy

\section{Introduction}

When polycrystalline material is plastically deforming, stress and strain heterogeneity fields are developing due to strain incompatibilities between grains of 2 different crystallographic orientations. Depending on the level of viscoplastic anisotropy of the material, the heterogeneity amplitude can be high.

The viscoplastic anisotropy of ice is known to be very strong, with dislocations gliding mostly on the basal plane with three equivalent $<11 \overline{2} 0>$ Burgers vector directions [1]. This results in strong kinematic hardening at grain boundaries and triple junctions [2]. As such ice is a good model for materials with high 31 viscoplastic anisotropy, such as magnesium [3, 4], 32 quartz [5] and olivine [6]. In ice, strong heterogeneity fields were measured by Digital Image Correlation 34 on polycrystalline samples deformed by compression

$$
24
$$
observed high stress and strain heterogeneities could only be correctly simulated providing a significant amount of non-basal slip activity in the corresponding areas, while the global non-basal activity remained low $[10,9]$. The same numerical constraint was derived for polycrystalline $\mathrm{Mg}$ in conditions where twinning was

March 29, 2018 
not activated [11]. However, up to now there exists no 85 unequivocal evidence for macroscopic strain resulting 86 from non-basal slip in ice $[12,13]$.

The presence of heterogeneous dislocation fields in experimentally and naturally deformed ice was mostly observed indirectly from substructures observations (Xray diffraction, optical analyses, EBSD...) $[8,9,14,15]$. In particular kink bands and double kink bands has been 90 commonly observed in polycrystalline ice deformed in the laboratory. The origin of these kink bands was indirectly interpreted to be related to some non-basal dislocation activity like climb or cross-slip $[8,9]$.

The objective of the present work is to couple both modeling and high resolution EBSD observations in order to extract a more direct causal relationship between stress and dislocation fields. These relationships are expected to be of particular significance in polycrystalline materials composed of phases exhibiting a 100 strong viscoplastic anisotropy.

\section{Experiment and EBSD analyses}

Both modeling and experimental analyses were per- 107 formed on the basis of compression creep experiments on samples made of laboratory grown polycrystalline ice with columnar grain shape [9]. Tests were stopped 110 at $1 \%$ strain in order to prevent the initiation of recrys- 111 tallization processes. A constant $0.5 \mathrm{MPa}$ stress was 112 applied at $-10^{\circ} \mathrm{C}$, with columns perpendicular to the ${ }_{113}$ compression direction (see [7] for details). Grain size of 114 the order of $1 \mathrm{~cm}$ enabled high resolution observations 115 in terms of microstructure and crystal orientation 116 variations at grain boundaries, triple junctions and grain 117 interiors. The deformed microstructure is shown in Fig. 118 1. In all analyses the same XYZ sample coordinate 119 system is used where $\mathrm{Z}$ is out of plane and the $\mathrm{Y}_{120}$ direction is parallel to the main compression axis. ${ }_{121}$ EBSD analysis was performed on a Philips XL-30- 122 ESEM-FEG at the Department of Geological Sciences 123 at Stockholm University. EBSD patterns were auto- 124 indexed using the CHANNEL 5 software of OI-HKL ${ }_{125}$ Technology. Samples analysed were extracted from 126 the deformed sample. They were uncoated, kept at ${ }_{127}$ high vaccum and frozen to the pre-tilted sample holder ${ }_{128}$ sledge cooled to temperature of -90 to $-100^{\circ} \mathrm{C}$ using 129 a cold stage (for more details see [8]). Data were 130 collected by moving the beam across a rectangular 131 area at steps of $15 \mu \mathrm{m}$. Noise reduction was performed ${ }_{132}$ following $[16,17]$. Accuracy of EBSD data is within 133 $0.3^{\circ}-0.4^{\circ}$ [18]. Fig. 1 presents selected EBSD maps 134 showing the local variations in orientations.

\section{Weighted Burgers Vector analysis}

To quantitatively analyze the EBSD point grid data we utilized the Weighted Burgers Vector (WBV) analysis explained in details in [19]. The WBV is a recently developed new quantity to constrain dislocation densities and dislocation types using EBSD data on two dimensional sections through crystalline materials. The WBV is defined as the sum, over all dislocation types, of [(density of intersections of dislocation lines with a map) $\times$ (Burgers vector)] and as such can be calculated from a planar set of orientation measurements such as in an EBSD orientation map. There is no assumption about the orientation gradient in the third dimension. The magnitude of the WBV gives a lower bound of the magnitude of the dislocation density tensor. The direction of the WBV can be used to constrain the types of Burgers vectors of the geometrically necessary dislocations present in the microstructure and their geometric relationship to intra-grain structures, for example subgrain walls. The WBV can then be decomposed in terms of the 3 main lattice vectors [20]. In the case of ice, the two a-axis lattice vectors are further decomposed into the 3 equivalent a-axis lattice vectors. We can calculate the net Burgers vector content of dislocations intersecting a given area of a map by an integration around the edge of this area. This integral WBV method is fast, complements point-by-point WBV calculations and, thanks to this integration, reduces the effect of noise on the analysis. A lower bound of the density of geometrically necessary dislocations can be estimated from this calculation. This estimation is not absolute but can be used for comparison purposes. It should be noted that the net Burgers vector value derived using the integral WBV method is sensitive to the chosen area both in terms of size and location.

WBV analysis data is represented in color coded maps showing the WBV magnitude, WBV directions as projected arrows onto the color coded maps and in pole figures with both the sample coordinate system and the crystal coordinate system (see Fig. 2).

Table 1 presents the integral WBV calculated over selected areas in the analyzed samples (Fig. 2 and Fig. 3), decomposed into the three a-axes and the c-axis of the ice crystallographic structure. Areas were selected to represent the observed spectrum of different microstructures present within the analyzed sample. Such microstructures include triple junctions, grain boundaries with or without asperities, areas in close vicinity or at 
distance to grain boundaries. Data from Table 1 shows 185 that the integral WBV values vary significantly depend- 186 ing on microstructure type and location. However, for 187 each selected area, we obtained several WBV values 188 and chose to present here values that are representative 189 for the selected area.

The accuracy of the integral WBV is dependent on the 191 angular resolution of the EBSD data. Using EBSD 192 data with high angular resolution (here within 0.3 de- 193 grees), we consider an integral WBV ratio of one spe- 194 cific Burger vector over the maximum WBV value of 195 0.5 significant.

\section{Local stress field estimation}

Full-field numerical simulations were performed 200 using the CraFT code as presented in [21]. The code is 201 based on the FFT method initially proposed in [22, 23], 202 extended to elasto-viscoplastic composites using a 203 step-by-step integration in time in [24] (see also the 204 numerical details in [21]). The method used in the 205 CraFT code finds a strain rate field associated with a 206 kinematically admissible velocity field that minimizes 207 the average local work rate under the compatibility and 208 equilibrium constraints. An iterative scheme is used 209 following a fixed point approach. It is numerically 210 more efficient than the finite element method [25], but is limited to simulations with microstructures with periodic boundary conditions.

The specimen undeformed microstructure was dis- 213 cretized into $512 \times 512$ Fourier points with a single ${ }_{214}$ layer of Fourier points in the third (Z) direction, 215 assuming thus infinite column length. To represent the ${ }_{216}$ microstructure, each Fourier point is allocated a c-axis 217 orientation according to the measured orientation of ${ }_{218}$ the underformed experimental sample. Consequently 219 grain boundary are not specifically defined as discrete 220 objects with specific physical characteristics other than ${ }_{221}$ a change in crystal orientation. Throughout the numeri- 222 cal simulations, no crystallographic orientation changes 223 are imposed, thus the microstructure (orientation, grain ${ }_{224}$ boundaries) does not evolve.

Creep conditions equivalent to the experimental ones were applied (constant $0.5 \mathrm{MPa}$ stress in the vertical 225 direction, transient creep up to $1 \%$ macroscopic strain).

The exact experimental boundary conditions (stress free 226 lateral surface) could not be reproduced due to the nu- 227 merical periodicity constraint. therefore, Accordingly, 228 stress and strain fields predicted close to the specimen 229 edges are not expected to be very accurate, however 230 Grennerat et al. [7] showed a limited impact on the 231 macroscopic response and estimated fields, especially 232 in the center of the modeled microstructure, where the areas of interest here are located.

The elasto-viscoplastic response of ice was modeled following the law and hypothesis discussed in details in [21]. To model ice, the used crystal plasticity formulation accounts for three different families of slip systems, namely the basal, prismatic and pyramidal systems, the latter two being taken stiffer than basal slip. A power law is considered for the evolution of the shear strain rate as a function of the resolved shear stress on each slip system that integrates kinematic hardening, slip system interactions and their evolution with time during transient creep. The material parameters of the law were determined by adjusting them according to experimental data available for single crystals as well as for polycrystals, as detailed in [21]. The Critical Resolved Shear Stress (CRSS) on each slip system was allowed to evolve with strain between initial and stationary values. Table 2 provides the relative CRSS for each slip system family, and the value of the stress exponent attributed to each family. It is worth noting that the relative CRSS is much higher for the pyramidal family, and that there is a relative softening of basal systems during transient creep [21].

The local heterogeneities of the strain and stress fields are illustrated in Fig. 4a) and b) where the spatial variation of the equivalent stress $\left(\sigma_{e q}=\sqrt{\frac{3}{2} \sigma_{i j} \sigma_{i j}}\right)$ and equivalent strain $\left(\varepsilon_{e q}=\sqrt{\frac{3}{2} \varepsilon_{i j} \varepsilon_{i j}}\right)$ are represented, respectively. For each simulated pixel of the microstructure, the deviatoric part of the stress tensor was decomposed into its eigenvector frame. From this decomposition, we extracted the eigenvector corresponding to the largest absolute eigenvalue. We built a composite vector representation based on the in-plane projection of a vector having the direction of this eigenvector, and the amplitude of the associated eigenvalue. This representation is given in Fig. 4c) for the area of interest, superimposed on the equivalent stress contour plot.

\section{Discussion}

Numerical modeling shows that local stress concentrations and stress field heterogeneities occur close to triple junctions and grain boundaries. The higher the mismatch between crystallographic grain orientations, the higher the concentration and heterogeneities (Fig. 4). For example, the triple junctions between grains that are similarly oriented show less stress and strain 
heterogeneities (between grains 1, 4 and 5) than that 285 between differently oriented grains (grains 1, 2 and 3). $\quad 286$ In addition, the relative orientation of grain boundary to 287 grain orientation influences the stress distribution. For 288 example, at the boundary between grains 1 and grain 4289 we observe a zone of high stress (Fig. 4c) where there 290 is a change in the grain boundary orientation relative to 291 the grain orientations.

Similarly, WBV analyses show that commonly WBV 293 magnitude associated with subgrain wall is highest 294 close to the grain boundary and decreases towards the 295 grain centre (Fig. 2).

Although the resolution of the model microstructure 297 is not as high as the one measured, there exists a 298 close correlation between the characteristics of the 299 stress heterogeneities (amplitude and direction of stress 300 field), and the amplitude and location of the lattice 301 misorientations measured by EBSD (see Fig. 3). This 302 is pronounced in areas close to triple junction, but also 303 apparent within the grain interior as shown in map G1, 304 Fig. 3 where the heterogenous stress band crossing 305 grain 1 correlates well with the observed subgrain 306 boundary crossing the corresponding EBSD map. In 307 general, areas which show high stress in simulation 308 are also characterized by high net Burger vector values 309 (e.g. MapG1 areaA, MapG4-1-5 areaE).

Areas close to triple junctions present WBV dominated ${ }_{311}$ by one a-axis direction or a combination of two a-axes. 312 This is illustrated by the decomposition of the integral 313 WBV data (Table 1) and represented in the inverse pole 314 figure plots in Fig. 3 (see for instance mapG1-3 areas A 315 and B and mapG4-1-5 area B, Table 1).

As illustrated by the inverse pole figure of subarea D 317 from map G4-1-5, there is a systematic presence of a 318 minor but significant c-axis component close to triple ${ }_{319}$ junctions. Such a c-axis component is considered as 320 non-negligible when the ratio $K_{c} / \max \left(K_{a i}\right)$ is higher 321 than 0.5 (see Table 1). In contrast, most of the areas ${ }_{322}$ analyzed in a grain interior show a c-axis component of ${ }_{323}$ the WBV which is very close to the detection limit (see 324 Table 1). Close to triple junctions, we observe near straight 326 subgrain boundaries. Their traces are parallel to the 327 c-axis, and the WBV are predominantly oriented along ${ }_{328}$ the a-axis perpendicular to the subgrain boundary trace ${ }_{329}$ (Fig. 1 and Fig. 3). The other a-axis contribution is 330 related to a continuous crystal bending perpendicular to 331 the c-axis (see Fig. 5 with a sketch of the bending axis). $\quad 332$ Similar observations were made in $[8,9]$. These only slightly curved subgrain boundaries com- 334 monly occur in parallel pairs with the main WBV ${ }_{335}$ pointing in opposite directions (Fig. 3 mapG1-3 336 and mapG4-1-5, Table 1). The parallelism of the subgrain boundary traces, their orientation relative to the crystallographic axes of the host grain together with the orientation of the main WBV a-axis component is consistent with a strongly crystallographically determined boundary development. The opposing directions of WBV in pairs of subgrain boundaries (Fig. 3) is consistent with kink bands with alternating opposing dislocation structures [26, 27, 28]. These crystallographically well defined kink boundaries could appear similar to the twinning modes in magnesium in the way that they both accommodate shear stress that cannot be resolved by the easy slip system i.e. basal slip in ice [3, 4, 29]. In metals, kink bands can occur at large deformation as strain localization modes, as predicted by the bifurcation analysis of [30], but also at the early stage of deformation in the presence of obstacles [31]. Chang et al. [31] used Dislocation Dynamic simulation to illustrate the stable position of an edge dislocation within the stress field of an alignment of edge dislocations forming the kink band. Consequently, in contrast to what was initially thought [9], climb or cross-slip do not seem to be necessary mechanisms to explain kink band formation. In summary, observations suggest that kink bands form in areas of very high local stress fields originating either from strain incompatibilities developed in materials with high viscoplastic anistropies or from high strain rates.

It should be noted that the used numerical model does not account for specific grain boundary properties. The question of the strain continuity at an interface such as grain boundary is therefore not addressed. In contrast, recent work [32] showed that stress accumulation at boundaries can explain grain boundary delamination in alloys. In the case of ice deforming during transient creep under low applied stress, no cracking was observed at grain boundaries, but a better account for continuity conditions of the plastic distortion at GB might be necessary to evaluate the impact of strain heterogeneities on recrystallization mechanisms for instance.

Our data show that in high stress concentration areas, close to triple junctions, a Burgers vector component parallel to c-axis is present. Cross slip of dislocations with Burgers vector lying in the basal plane cannot explain this; instead it could be interpreted by a local activity of a non-basal slip system. Conceptually, such a non-basal slip activity is expected only in such high stress areas, as non-basal activity requires high level of local stress in order to overcome the high critical resolved shear stress required for non-basal slip 
[1]. So far, the very few observations on non-basal 385 dislocation activity in ice were performed in conditions 386 with very few dislocations activated, that could hardly 387 be extrapolated. Indeed, they were performed using 388 X-ray diffraction topography which enable individual 389 dislocation observation at the very early stage of 390 deformation [33, 34].

The observed crystal bending parallel as well as per- 392 pendicular to the kink bands which is accommodated 393 by the shown presence of Burgers vectors along not 394 one but two a-axes in high stress areas, may results in 395 the development of subgrain boundaries both parallel 396 and perpendicular to c-axis. This then will result in 397 the formation of a subgrain with near perpendicular 398 subgrain boundaries, one parallel to c-axis and one 399 perpendicular to c-axis. Such a developing subgrain is shown in Fig. 5 and has been described in [8]. This subgrain boundary formation represents one of the re- 402 crystallization processes relaxing strain heterogeneities ${ }^{403}$ at macroscopic strain exceeding the transient creep ${ }_{404}^{404}$ regime $[2,35]$.

In summary:

- Coupled full field elasto-viscoplastic modelling ${ }^{410}$ and detailed EBSD analysis show the effect of ${ }_{412}^{41}$ stress heterogeneities (magnitude and orientation) 413 on the dislocation field.

- The WBV c-axis component measured in high stress areas could be consistent with local activation of non-basal slip.

- At low strain, formation of kink bands with a well ${ }_{421}$ defined crystallographic character appears as an ef- ${ }^{422}$ ficient accommodation deformation mode, similar to twinning in $\mathrm{Mg}$.

- Distinct misorientations across and perpendicular ${ }^{42}$ to kink boundaries form substructures which act as 428 precursors for grain nucleation.

- The approach presented is applicable to materials with significant viscoplastic anisotropy.

Acknowledgments: Financial support by French ANR is ${ }_{435}^{434}$ acknowledged (project ELVIS, \#ANR-08-BLAN-0138, 436 project DREAM \#ANR-13-BS09-0001). Together with ${ }^{437}$ support from institutes INSIS and INSU of CNRS, ${ }_{439}^{438}$ France. The authors gratefully acknowledge the ESF 440 Research Networking Programme Micro-Dynamics ${ }^{441}$ of Ice (www.microdice.eu). MM is part of Labex ${ }^{442}$ OSUG@2020 (ANR10 LABX56). SP acknowl- ${ }_{444}^{443}$ edges the financial support of the European Science 445
Foundation under the EUROCORES Programme, EuroMinSci, MinSubStrDyn, No. ERAS-CT-2003980409 of the European Commission, DG Research, FP6. SP acknowledges additional funding from ARC DP120102060 and FT1101100070. Funding by ARC Centre of Excellence for Core to Crust Fluid Systems (www.CCFS.mq.edu.au) allowed a Research visit of M.M. to Macquarie University. This is contribution 534 from the ARC Centre of Excellence for Core to Crust Fluid Systems (http://www.ccfs.mq.edu.au) and 975 in the GEMOC Key Centre. Funding from the Australian Research Council (ARC) through the CCFS Visiting Researcher scheme is gratefully acknowledged (www.GEMOC.mq.edu.au).

[1] Hondoh T. In: T Hondoh (Ed.), Physics of Ice Core Records. Sapporo: Hokkaido University Press, 2000, 2-34.

[2] Duval P, Ashby M, Anderman I. J Phys Chem 1983;87:4066.

[3] Barnett MR. Materials Science and Engineering: A 2007;464:1.

[4] Barnett MR. Materials Science and Engineering: A 2007;464:8.

[5] Hobbs BE. Preferred orientation in deformed metals and rocks, chapter The geological significance of microfabric analyses. Academic Press, Orlando, 1985, 463-484.

[6] Nicolas A, Poirier JP. Crystalline Plasticity and Solid State Flow in Metamorphic Rocks. John Wiley \& Sons, 1976.

[7] Grennerat F, Montagnat M, Castelnau O, Vacher P, Moulinec H, Suquet P, Duval P. Acta Materialia 2012;60:3655.

[8] Piazolo S, Montagnat M, Blackford JR. Journal of Microscopy 2008;230:509.

[9] Montagnat M, Blackford JR, Piazolo S, Arnaud L, Lebensohn RA. Earth and Planetary Science Letters 2011;305:153.

[10] Lebensohn RA, Montagnat M, Mansuy P, Duval P, Meyssonnier J, Philip A. Acta Materialia 2009;57:1405 .

[11] Martin G, Sinclair CW, Lebensohn RA. Materials Science and Engineering: A 2014;603:37.

[12] Shearwood C, Whitworth RW. Phil Mag 1991;A64:289.

[13] Louchet F. C R Physique 2004;5:687.

[14] Montagnat M, Duval P, Bastie P, Hamelin B, Lipenkov VY. Earth and Planetary Science Letters 2003;214:369.

[15] Donges J, Montagnat M, Bastie P, Grennerat F. Nuclear Instruments and Methods in Physics Research Section B: Beam Interactions with Materials and Atoms 2013;300:6 .

[16] Prior D. Journal of Microscopy 1999;195:217.

[17] Piazolo S, Bestmann M, Prior DJ, Spiers CJ. Tectonophysics 2006;427:55.

[18] Borthwick VE, Piazolo S. Journal of Structural Geology 2010; 32:982.

[19] Wheeler J, Mariani E, Piazolo S, Prior DJ, Trimby P, Drury MR. Journal of Microscopy 2009;233:482.

[20] Wenk RH, Bulakh A. Minerals: Their Constitution and Origin. Cambridge Univ. Press, 2004.

[21] Suquet P, Moulinec H, Castelnau O, Montagnat M, Lahellec N, Grennerat F, Duval P, Brenner R. Procedia IUTAM 2012;3:76.

[22] Moulinec H, Suquet P. Computer Methods in Applied Mechanics and Engineering 1998;157:69.

[23] Moulinec H, Suquet P. European Journal of Mechanics A/Solids 2003;22:751.

[24] Idiart MI, Moulinec H, Ponte Castañeda P, Suquet P. Journal of the Mechanics and Physics of Solids 2006;54:1029.

[25] Prakash A, Lebensohn RA. Modelling Simul Mater Sci Eng 2009;17:1. 
[26] Wilson C, Burg J, Mitchell J. Tectonophysics 1986;127:27.

[27] Nishikawa O, Takeshita T. Tectonophysics 1999;301:21 .

448 [28] Yamasaki M, Hagihara K, ichi Inoue S, Hadorn JP, Kawamura Y. Acta Materialia 2013;61:2065

[29] Chapuis A. Materials Science and Engineering: A 2014 590:401.

2 [30] Sedláček R, Blum W, Kratochvíl J, Forest S. Metallurgical and Materials Transactions A 2002;33:319.

[31] Chang HJ, Gaubert A, Fivel M, Berbenni S, Bouaziz O, Forest S. Computational Materials Science 2012;52:33.

[32] Messner MC, Beaudoin AJ, Dodds Jr RH. Engineering Fracture Mechanics 2015;133:70.

458 [33] Shearwood C, Withworth R. JGlaciol 1989;35:281.

459 [34] Shearwood C, Whitworth R. Acta Metall Mater 1993;41:205.

460 [35] Montagnat M, Durand G, Duval P. Supp Issue Low Temperature Science 2009;68:81. 


\begin{tabular}{lc|ccccccc}
\multicolumn{7}{c}{ Analyses areas } & \multicolumn{7}{c}{ Integral WBV $(\mu \mathrm{m})^{-2}$} & $\rho$ \\
\hline Map & gr/area & $\mathrm{K}_{a 1}$ & $\mathrm{~K}_{a 2}$ & $\mathrm{~K}_{a 3}$ & $\mathrm{~K}_{c}$ & $\mathrm{~K}_{c} / \max \left(\mathrm{K}_{a i}\right)$ & $\left(10^{10} \mathrm{~m}^{-2}\right)$ \\
\hline MapG2 & 2/A & -0.32 & 0.28 & 0.04 & 0.05 & 0.156 & 18.49 \\
& 2/B & 0.18 & -0.15 & -0.03 & 0.02 & 0.111 & 5.62 \\
MapG1 & 1/ A & 0.08 & -0.01 & -0.07 & -0.02 & 0.250 & 1.18 \\
& 1/B* & -0.01 & 0.01 & 0.01 & 0.01 & & 0.04 \\
\multirow{4}{*}{ MapG1-3 } & 1/A & -0.28 & 0.11 & 0.18 & 0.08 & 0.282 & 12.93 \\
& 1/B & 0.73 & -0.43 & -0.30 & -0.12 & 0.164 & 82.22 \\
\multirow{4}{*}{ MapG4-1-5 } & 3/C & 0.29 & -0.13 & -0.15 & -0.1 & 0.349 & 13.35 \\
& 1/A & -0.19 & 0.05 & 0.13 & 0.11 & 0.589 & 6.76 \\
& 1/B & 0.10 & -0.03 & -0.06 & 0.04 & 0.414 & 1.61 \\
& 4/C & 0.09 & -0.06 & -0.02 & -0.01 & 0.115 & 1.22 \\
& 4/D & 0.05 & -0.01 & -0.05 & -0.20 & 3.750 & 4.51 \\
& 5/E & 0.44 & -0.30 & -0.13 & -0.29 & 0.664 & 38.46 \\
\hline
\end{tabular}

Table 1: Integral WBV decomposed onto the three crystallographic axis of the ice crystal, for specific areas in EBSD maps shown in Figs. 1,2 and 3. The two Ka vector directions are symmetrically equivalent, they were decomposed into the 3 a-directions following [20]. The relative activity of $\mathrm{K}_{c} / \max \left(\mathrm{K}_{a i}\right)$ is also given. $\rho$ provides a lower bound estimate of the geometrically necessary dislocation density in the area. Step size is $15 \mu \mathrm{m}$ for all EBSD maps. * minimum value of the net Burgers vector magnitude. "gr" signifies grain number (cf. Fig. 1) and "area", subarea as shown in Figs. 2 and 3.

\begin{tabular}{l|c|c|l} 
System family & $\tau_{\text {iniR }}$ & $\tau_{\text {statR }}$ & $n_{s}$ \\
\hline basal & 1 & 1 & 2 \\
prismatic & 1.3 & 68.2 & 2.85 \\
pyramidal & 38.75 & 176 & 4
\end{tabular}

Table 2: Relative CRSS for the three slip system families at the beginning of the transient creep, $\tau_{\text {iniR }}$, and at the end, $\tau_{\text {stat }} . n_{s}$ is the stress exponent that was adjusted by [21] for each family. The absolute value of $\tau_{\text {ini }}$ for the basal slip systems was fitted at 0.1 by [21], and the absolute value of $\tau_{\text {stat }}$ for the basal slip systems was fitted at 0.022 . 


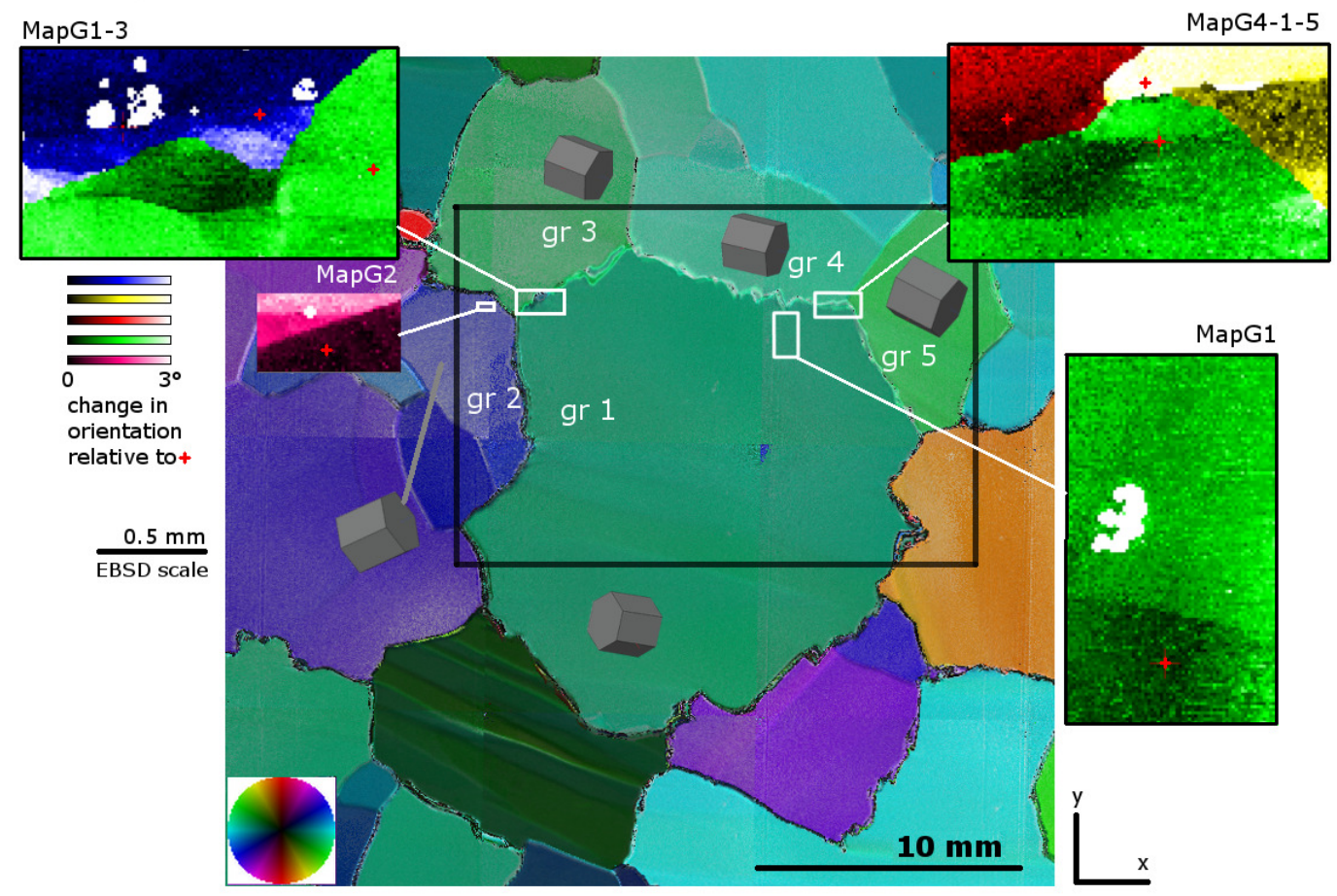

Figure 1: Microstructure of a selected area of the columnar ice sample (in the plane perpendicular to the columns) after compressive strain of $10 \%$ along y direction. The central microstructure is color coded according to the measured c-axis orientation as represented by the color wheel (inset). EBSD maps of selected areas (marked by white rectangles) are shown with color code for change in orientation according to color scheme provided on the left side of the central microstructure. Insets of 3D crystal orientations (grey hexagons) are provided by EBSD analyses. Grains are given numbers for ease of reference (e.g. gr 1). Note labeling of the selected areas for which EBSD maps were obtained corresponds to the grain numbers of the grains present in the respective map.

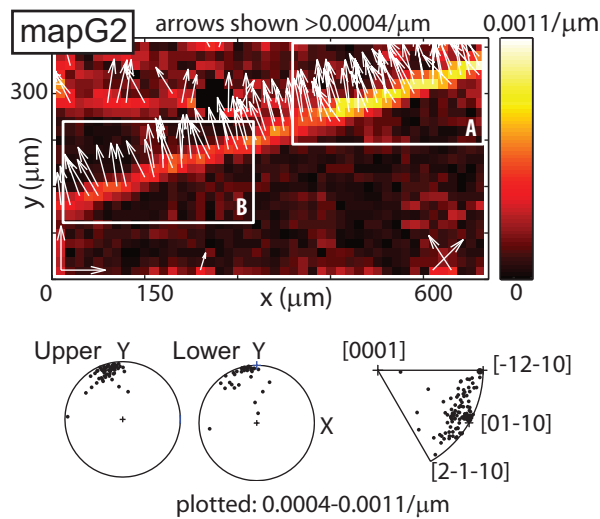

Figure 2: WBV magnitude (color range) for mapG2, WBV directions are shown as white arrows on map, above the threshold value for WBV magnitude of $0.0004 / \mu \mathrm{m}$, and on pole and inverse pole figures below. Areas analysed via integral WBV decomposition on crystallographic axes are marked as white boxes (A and B), corresponding data is given in Table 1. Note area A is in close vicinity to grain boundary. 


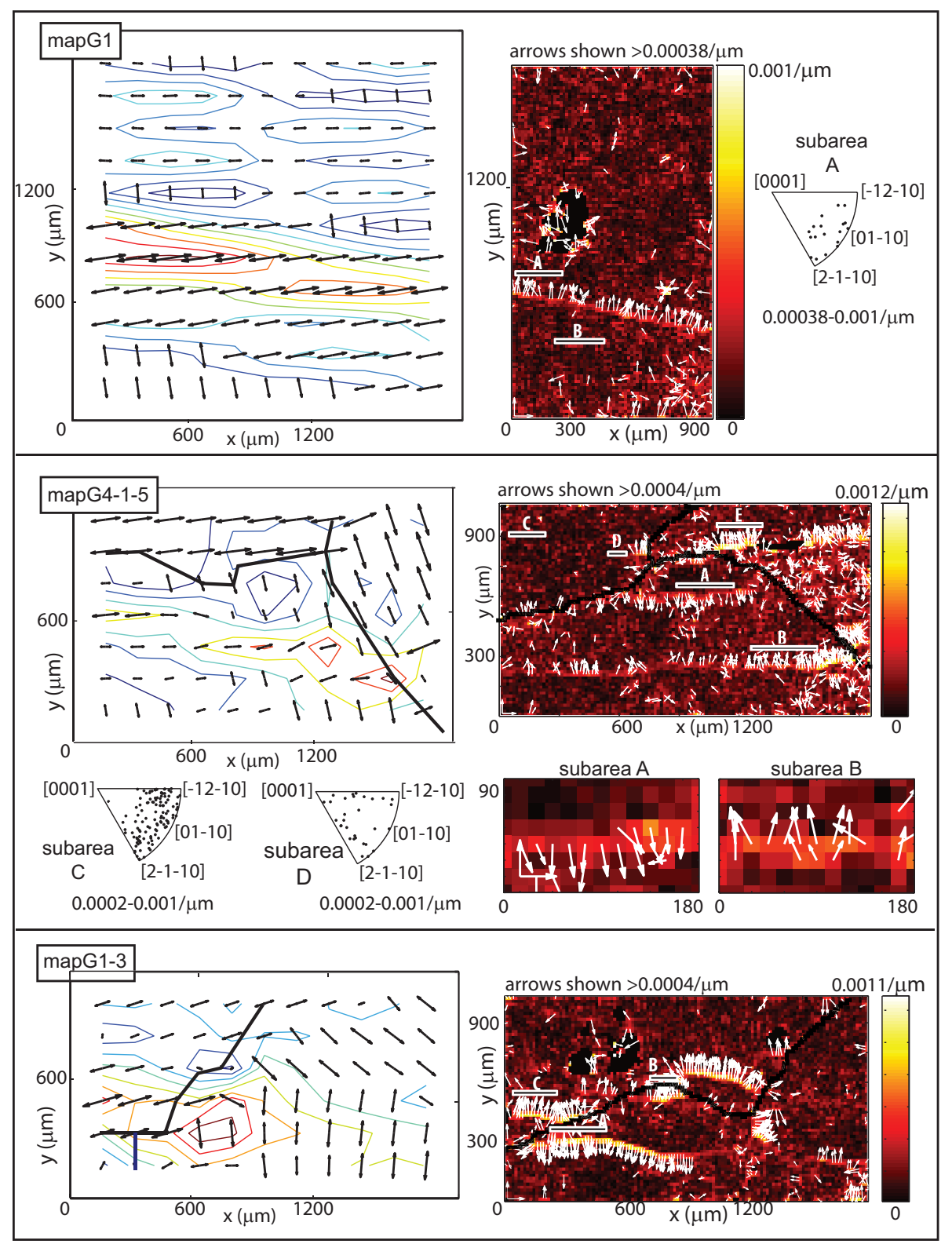

Figure 3: Comparison between stress field and dislocation field; deviatoric stress eigenvectors (left) zoomed in the areas selected for the WBV analyses (right maps). WBV analyses are shown with WBV magnitude (color range), WBV directions shown as white arrows on map (over a threshold value), and on inverse pole figures for selected areas (labeled A to D). Only upper limits are shown for these areas. Integral WBV of subareas are given in Table 1. Black lines represent grain boundaries. Due to limitation in the simulation configuration (see text), the correspondence between modeled and observed microstructures such as the position of grain boundaries is approximate. 


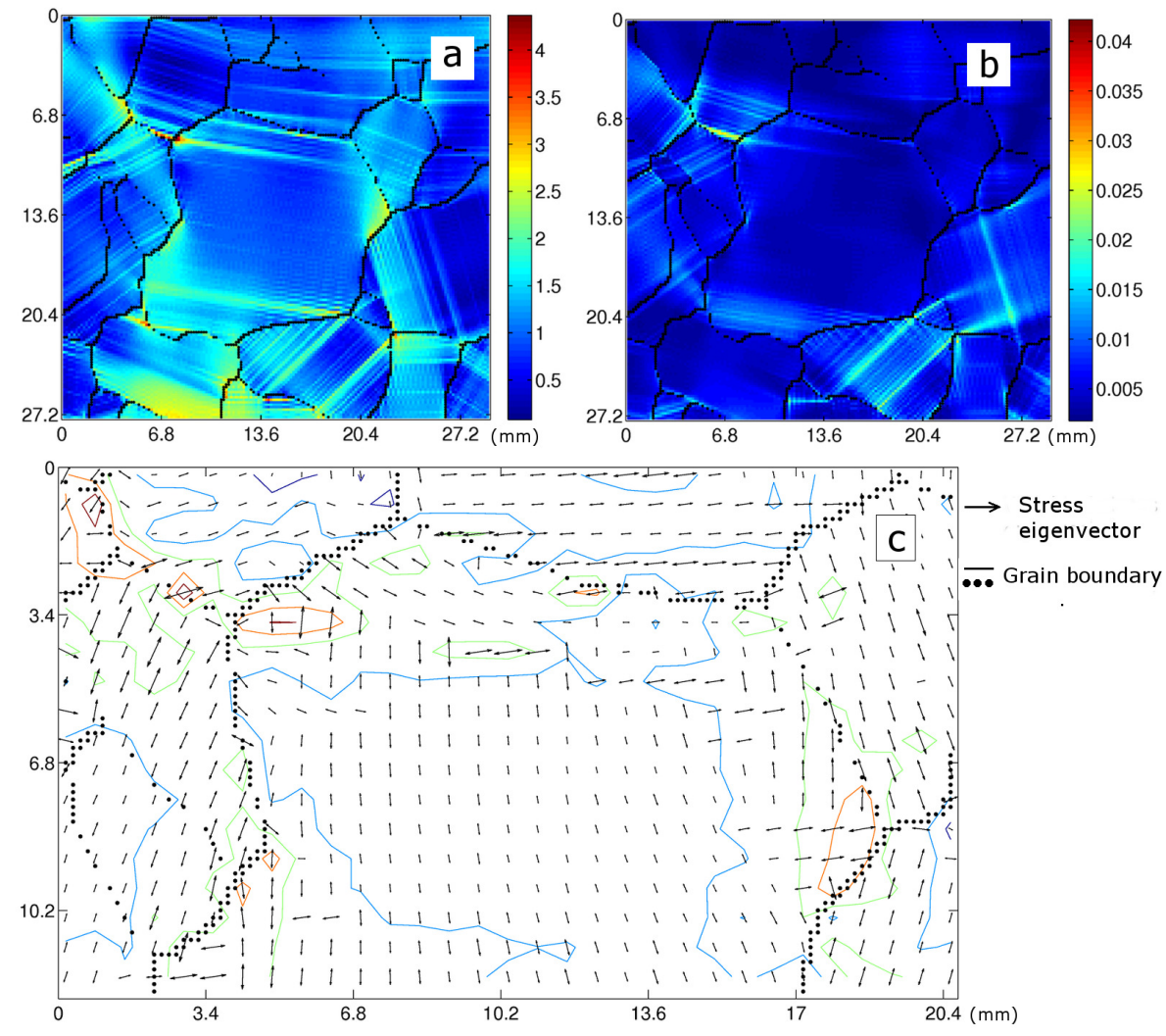

Figure 4: Maps of equivalent stress (in MPa) (a) and of equivalent strain (b) predicted by the full-field simulation using CraFT on the area of interest after 0.01 macroscopic strain under compression creep. c) provides a map of the deviatoric stress eigenvectors projected on the plane, superimposed on the equivalent stress contour plot (same scale as in a)). Grain boundary traces are superimposed for clarity but they are not physical entities in the code.
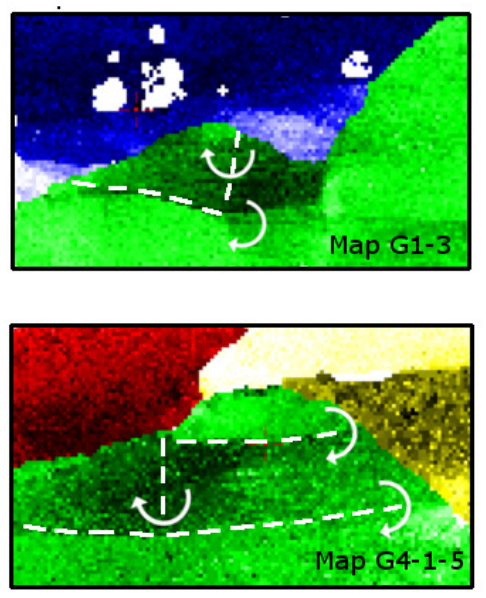

Figure 5: Planes of crystal bending (white stippled lines) for subgrain boundaries and associated rotation directions. The nearly horizontal subgrain boundaries are associated with abrupt misorientations, while the vertical ones correspond to more progressive misorientations. Both sub-structures were quantified in term of WBV, see Fig. 3. 